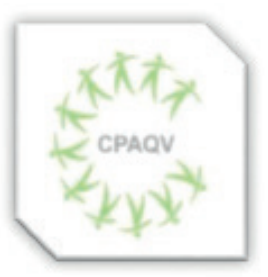

ISSN: 2178-7514
ARTIGO DE REVISÃO

\section{EDUCAÇÃO FÍSICA ESCOLAR E OS EFEITOS NAS VARIAÇÕES DE HUMOR DOS ESTUDANTES - UMA REVISÃO NARRATIVA}

\section{School physical education and the effects on students' mood variations - A Narrative Review}

Luana Marcela Ferreira Campanhã ${ }^{1}$, Maria Luiza Miranda Paulino da Silva ${ }^{1}$, Ademir Testa Junior ${ }^{2,3}$, Renan Floret Turini Claro ${ }^{2}$, Gabriel de Souza Zanini ${ }^{1,2}$

Vol. 13 | No. 2 | Ano 2021

\title{
RESUMO
}

As atividades atreladas a educação física escolar e suas influências no humor dos escolares pode ser considerada na literatura como um ponto positivo e atual, apesar da escassez de estudos sobre sua relação com as variáveis psicológicas dos indivíduos em idade escolar. A seguinte pesquisa teve como objetivo levantar estudos sobre a ligação entre as atividades físicas na escola e sua correlação com o humor das crianças e jovens que participam dessas atividades. Os achados demonstram que a prática regular de atividades físicas pode não apenas melhorar a questão comportamental de estudantes, como também aumentar significativamente seu engajamento nos processos de ensino e aprendizagem, sendo, portanto, um aspecto impar para a promoção de saúde, bem como para a melhora da performance acadêmica. Os estudos revisados destacam as atividades físicas como um ponto muito importante para a qualidade de vida dos alunos, assim como a importância da abordagem dos educadores para com seus educandos e a ludicidade como uma prática indispensável para o desenvolvimento das crianças. Todavia, mais pesquisas são necessárias para o aprofundamento do tema.

Palavras-chave: Educação Física escolar, sociabilidade, saúde mental, atividade física, profissional de educação física, atividades lúdicas.

\begin{abstract}
Activities linked to physical education at school and its influence on the mood of schoolchildren can be considered in the literature as a positive and current point, despite the scarcity of studies on their relationship with the psychological variables of school-age individuals. The following research aimed to raise studies on the link between physical activities at school and its correlation with the mood of children and young people who participate in these activities. The findings demonstrate that the regular practice of physical activities can not only improve the behavioral issue of students, but also increase their engagement in the teaching and learning processes, being, therefore, a unique aspect for health promotion, as well as for improvement. of academic performance. The reviewed studies stand out as physical activities as a very important point for the quality of life of students, as well as the importance of the approach of educators to their students and playfulness as an essential practice for the development of children. However, more research is required to deepen the topic.
\end{abstract}

Keywords: School Physical Education, sociability, mental health, physical activity, physical education professional, recreational activities.

\footnotetext{
1 Departamento de Educação Física, Universidade Estadual Paulista (UNESP)

2 Departamento de Educação Física, Faculdades Integradas de Jaú (FIJ)

3 Escola de Artes, Ciências e Humanidades, Universidade São Paulo
}

Autor de correspondência

Gabriel de Souza Zanini - gabriel.zanini@unesp.br 


\section{INTRODUÇÃO}

Atualmente, dentro do contexto de ensino professores de educação física possuem dificuldades para abordar e entender as variações de humor em estudantes, e, consequentemente não possuem uma estratégia para desempenhar seu trabalho de maneira mais coerente, por muitas vezes negligenciando aspectos relacionado ao comportamento dos alunos. Ramires et $\mathrm{al}^{(1)}$ demonstraram que crianças em idade escolar tendem a manifestar sintomas psicopatológicos que não são identificados e não recebem o devido atendimento, corroborando com a falta de preparo por parte dos professores em identificar estes fenômenos.

A dificuldade em identificar eventos comportamentais ou psicopatológicos atrapalha os processos de aprendizagem em diversos contextos, como o ensino escolar, ademais durante esse período de desenvolvimento. Além disso, variáveis psicológicas relacionam-se a inúmeras dificuldades em sociabilidade dificultando a interação entre estudantes, e , também dentro do âmbito familiar, o que promove uma redução da participação de atividades escolares e nos demais ambientes sociais ${ }^{(2)}$.

Deste modo desordens comportamentais e psicológicas podem ocasionar inúmeros problemas de sociabilidade, e, localizar e identificar essas variáveis podem ser de suma importância para que o entendimento desses aspectos psicológicos, possam contribuir positivamente para o desenvolvimento social desses estudantes, abrangendo também a melhor forma de os inserir em todos os espaços para que não haja a exclusão dos mesmos, tanto em um contexto de aprendizagem quanto de sociabilização ${ }^{(3)}$.
Ademais, a partir desta percepção, os professores poderiam identificar a ocorrência do estabelecimento de padrões comportamentais nos estudantes, que, por sua vez, poderia levalos a um melhor planejamento e estratégias de ensino visando o melhor estabelecimento das relações e emoções destes estudantes ${ }^{(4)}$. Deste modo, dar a devida atenção para a saúde mental dos alunos é de suma importância, haja vista a grande complexidade que existe nas relações durante os anos de formação, levando em conta as abordagens de ensino utilizadas, os vínculos entre alunos e professores. Neste contexto o professor de educação física pode ter um papel primordial em identificar estes fenômenos dado o contexto mais socializador de suas aulas.

Além disso, sabe-se que um maior nível de atividade física está associada a uma melhora nos padrões comportamentais ${ }^{(4-6)}$, assim, as aulas de educação física tornam-se um ponto fundamental e indispensável para que os alunos se desenvolvam e tenham uma melhora em suas variáveis físicas, psicológicas e sociais ${ }^{(7)}$. Em um estudo realizado por Ferreira et al ${ }^{(8)}$ verificou-se que existe uma relação positiva entre a prática de exercícios físicos e lazer e o bom desempenho acadêmico em estudantes do ensino fundamental e médio. Os resultados do estudo sugerem que a prática esportiva, as aulas de educação física escolar e a atividade física durante o lazer possuem uma relação intrínseca com a saúde mental dos jovens ${ }^{(8)}$.

A partir do panorama apresentado este estudo teve como objetivo descrever os principais efeitos da prática de atividades físicas nas variáveis comportamentais e sociais em crianças com idade escolar. 


\section{MÉTODOS}

\section{Estratégia de Busca e Seleção dos Estudos}

O estudo trata-se de uma revisão narrativa, a qual foi elaborada a partir das seguintes etapas: 1) definição da temática e questão do estudo; 2) seleção das bases de dados; 3) definição dos dados prioritários para posterior análise; 4) avaliações e filtragens refinadas dos estudos e resultados encontrados e 5) síntese descritiva dos principais $\operatorname{achados}{ }^{(9)}$.

Para a realização deste estudo de revisão narrativa, foi feito um levantamento bibliográfico no mês de junho de 2021 na base virtual de dados Medline via PubMed por dois autores independentes. $\mathrm{O}$ protocolo utilizado para a busca dos estudos foi feito por meio da seleção de palavras chaves previamente definidas (isoladas e/ou combinadas) na língua inglesa e língua portuguesa, no qual foram utilizados os seguintes termos de indexação e termos meSH: Educação Física Escolar (School physical education), Saúde mental de jovens e crianças (Mental health of young people and children), Aulas de Educação Física e Socialização (Physical education classes and sociability), Atividades Recreativas (Reacreative Activities), Assistencia na Saúde de Jovens e Crianças (Assistance in health of children and young people). Dessa forma, foi questionada a influência da educação física na saúde mental dos escolares, e como o profissional de educação física pode auxiliar na melhora psicopatológica de seus alunos, com atividades físicas e lúdicas. A Figura 1 retrata o fluxograma para a obtenção dos estudos analisados.

Figura 1: Fluxograma da revisão narrativa no formato PRISMA

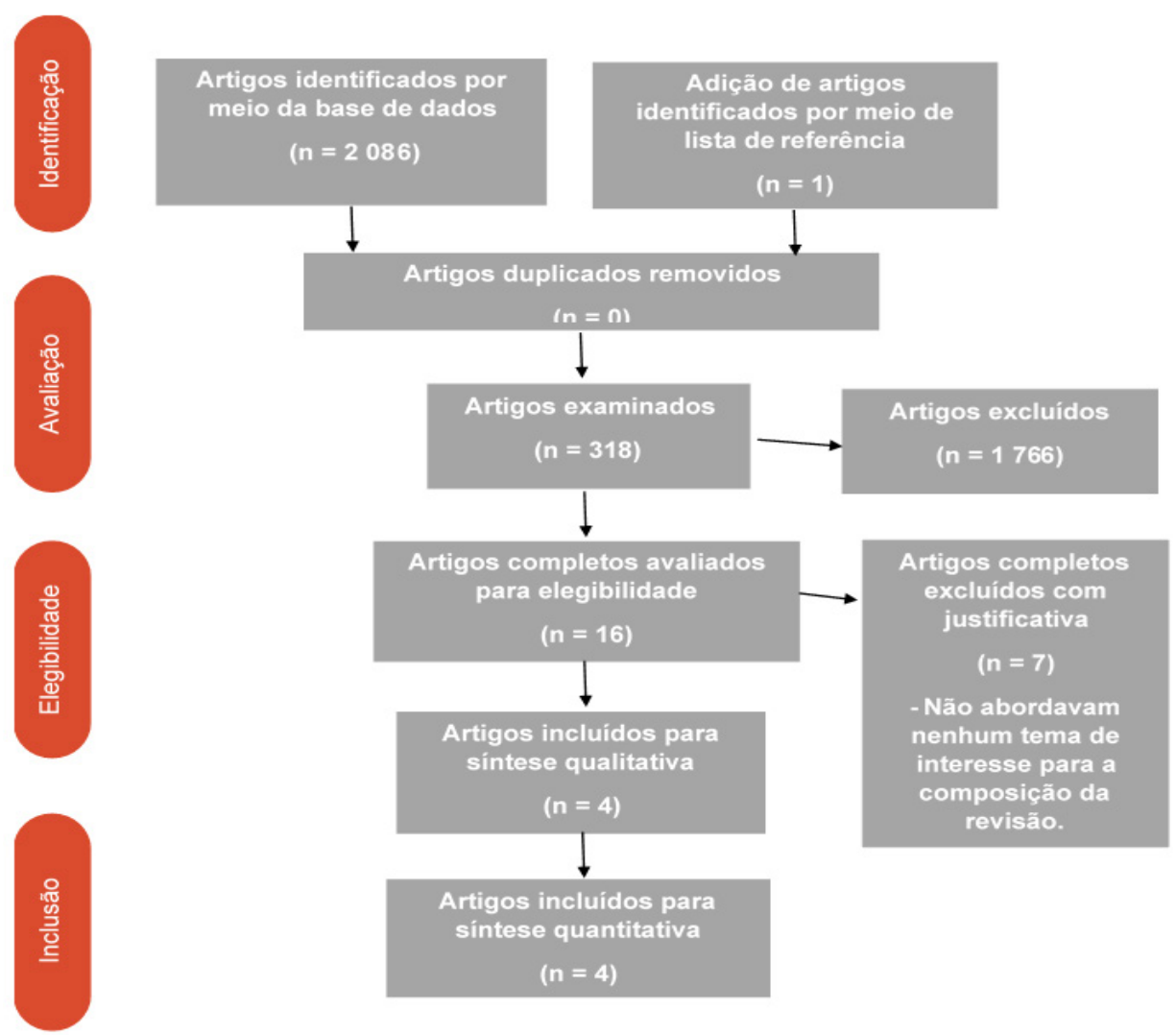




\section{RESULTADOS}

Beneficios do exercicio físico para o corpo e o psicológico das crianças e jovens

A atividade física moderada e vigorosa possui um papel muito importante para o controle da obesidade, contudo, também é fundamental na melhora do humor auxiliando assim os indivíduos a lidarem com vários tipos de estressores. Existe uma enorme associação entre a atividade física e a produtividade, indivíduos que praticam atividades físicas regularmente tendem a sentir níveis mais consideráveis de bem-estar psicológico e são mais produtivos do que outros que não possuem esse hábito. E neste contexto, a educação física tem um papel fundamental nas escolas de promover essa vida ativa e proporcionar a participação desses indivíduos em atividades físicas ao longo da vida adulta ${ }^{(10)}$.

No estudo de Kawabata et al (10) foi observado que utilizando a teoria do comportamento planejado e voltados para a motivação as intervenções tendem a trazer maiores benefícios, pois ações fundamentadas podem gerar alterações mais significativas nos níveis de atividade física a longo prazo. Neste sentido, os autores empregaram este modelo de incentivo para uma maior aderência para a prática de atividades físicas por parte dos estudantes. Os estudos atrelados a esta teoria trazem dois fatores para a validação das intervenções propostas aos estudantes, o primeiro fator se caracteriza como a saliência da informação e o segundo os tipos de crenças e barreiras comunicadas durante o período de intervenção.

Neste sentido pode-se compreender que as cobranças e interações entre a instituição (escola) e os estudantes tornam-se mais positivas à medida que informações mais importantes eram transmitidas. Os tipos de crenças e barreiras comunicadas durante o período de intervenção podem ter como alvo as crenças comportamentais, ressaltando benefícios salientes também controlando a ênfase das desvantagens relacionadas às atividades físicas. Como outra alternativa, as intervenções podem ter como alvo, crenças normativas que frisam as atitudes de outras pessoas importantes para a vida dos estudantes, como por exemplo as atitudes dos professores ou pais em relação à atividade física $^{(10)}$.

Em um estudo realizado por Smedegaard et al. ${ }^{(1)}$ enfatiza que atividades físicas praticadas regularmente contribuem para a melhora nas habilidades sociais e na autoestima de crianças e jovens, sendo o mesmo, um ponto muito positivo para o bem estar geral desses indivíduos. E nesse estudo, similarmente ao anterior, o nível observado de atividade física não é o ideal para atingir os efeitos significantes. Como alternativa, intervenções em escolas foram coordenadas e esses estudos mostraram que as atividades escolares estão associadas a melhor saúde física, psicológica e social.

Além disso, um ponto importante observado no estudo de Smedegaard et al. ${ }^{(11)}$ 
são as experiências positivas com os educadores que levaram ao bem-estar social mais elevado. No mesmo contexto é possível que os fatores de saliência da informação e os tipos de crenças e barreiras comunicadas durante o período de intervenção que foram abordados acima pelos autores Kawabata et al ${ }^{(10)}$, possam trazer um nível de bem estar e conforto importante para os estudantes se sentirem atraídos para as atividades que serão ministradas.

Elsborg et al. ${ }^{(12)}$, demonstraram que o fato do indivíduo ter um estilo de vida mais saudável pode estar relacionado a diversos fatores, que pode influenciar a qualidade de vida das pessoas quando pensamos na saúde física, mental, psicológica e social é o fator socioeconômico, tendo em vista que boa parte da população em países de baixa renda se encontram em localidades socioeconomicamente desfavorecidas, possivelmente ocasionando uma redução na expectativa de vida. E podemos pensar em projetos relacionados a atividades físicas, pois os ganhos para a saúde mental e a prevenção de doenças não transmissíveis podem ser de grande significância para essa população, principalmente quando é levado em conta o fato de que a prática de atividade física pode ser de grande acessibilidade para essas pessoas que se encontram à margem da sociedade, quando relacionadas a promoção da saúde especificamente para essa população.

No estudo apresentado por Lubans et al. (5) sobre os mecanismos psicossociais indicam uma correlação entre auto percepções físicas e indicadores de bem-estar, uma grande parte dos estudos retratam que as percepções dos indivíduos por eles mesmos estão efetivamente relacionadas ao mal-estar na juventude. Os indicadores neurobiológicos como destacado na revisão são estudos de uma vertente mais atual, dessa maneira, esse campo cognitivo é de difícil compreensão. Mas, existem indícios dos estudos apresentados de que a atividade física possui influência sobre o desempenho cognitivo. Um exemplo abordado foi uma intervenção de atividade física realizada num período de nove meses que tinha por objetivo melhorar a aptidão cardiorrespiratória, a qual resultou em um melhor desempenho, como também aumentou a atenção dos indivíduos em algumas tarefas que requerem inibição melhorada e flexibilidade cognitiva.

Neste sentido Poirel (6), salienta que os problemas com a saúde mental se tornaram uma grande dificuldade para a saúde pública, e a maneira mais simples, acessível e prazerosa de lidar com esse empecilho são as práticas regulares de atividades físicas.

A influência da atividade lúdica e a sua importância para o desenvolvimento das emoções.

No contexto educacional o professor é uma peça fundamental para a formação de seus alunos e futuros adultos em meio a uma sociedade e, tendo isso em mente Prodócimo ${ }^{(13,14)}$ afirma que um educador necessita ser empático e afetivo para conseguir transmitir aos estudantes o que são seus sentimentos e suas emoções. A todos 
os momentos emoções estão conosco e elas podem nos influenciar diretamente, entendê-las e aprender a lidar com esses sentimentos são passos muito importantes para o desenvolvimento de um indivíduo.

É no ambiente escolar que o professor pode trabalhar essas manifestações, pois, este espaço é complementar a vida das crianças por um longo período. O jogo e a brincadeira podem abordar de inúmeras formas as emoções, visto que, vitória ou derrota levam a emoções distintas que podem ser complexas, pode-se elaborar diversas formas de lidar com as situações junto aos estudantes ${ }^{(13)}$.

Conseguimos entender o jogo e a brincadeira como possibilidades que os estudantes têm de entender suas emoções, neste contexto Prodócimo ${ }^{(13)}$, se apoia na observação das manifestações emocionais de cinco escolas diferentes, sendo elas públicas e particulares, e algumas emoções foram escolhidas para serem observadas por serem caracterizadas como emoções básicas, elas são alegria, raiva, companhia/amor, medo, asco e tristeza. Como já foi mencionado, as emoções podem se manifestar em diferentes momentos e de diferentes formas, isso abre espaço para o aprendizado dos estudantes de como agir perante seu estado de humor naquele momento, por exemplo, é normal sentir raiva por perder ou não saber executar algum movimento, mas partir para agressão ou ofender algum colega não pode ser normalizado pelo professor, são nessas janelas que o educador deve agir e mostrar como o estudante pode resolver de uma maneira socialmente adequada.

Podemos entender a educação física como uma grande gama de possibilidades de relações sociais que ocorrem a partir das atividades que são apresentadas pelos professores. Essas relações sociais podem ser extremamente saudáveis para os estudantes, mas por outro lado não tem como fingir que essas relações muitas das vezes agressivas não estão acontecendo nas escolas. Cabe ao professor intervir quando essas manifestações de agressividade ocorrerem, independente do grau que seja, o professor está ali para lidar com essas situações que não fazem parte do planejamento, por mais inesperado que sejam essas situações cabe ao educador agir para que parte do processo educativo dessas crianças também possa estar voltado para o respeito ao próximo $^{(14)}$.

O tema emoções e sentimentos geralmente é deixado de lado pelas escolas, isso ocorre por conta do modelo que as escolas se fundamentam, o apreço pelo ensino imobilizado, um bom aprendizado não está apenas a imobilidade dos estudantes, que são obrigados a ficarem sentados por muitas horas em suas cadeiras, a alegria expressa por eles em apenas sair da sala de aula já diz muito. O lúdico não deve ser posto apenas como um passatempo, ele é muito mais importante para o desenvolvimento do que apenas para o controle de uma turma entediada ${ }^{(13)}$. 
Os estados de humor apresentados por crianças e jovens afetam diretamente o seu aprendizado. $\mathrm{O}$ aprendizado delas se torna muito mais leve e fácil quando se sentem motivados e seguros com o ambiente onde estão inseridos, com este espaço eles têm a oportunidade de sentir um bem-estar muito maior e consequentemente uma melhor aprendizagem ${ }^{(13)}$.

Em outro estudo vemos a importância de atividades recreativas fora do ambiente escolar, aqui Elsborg et al. ${ }^{(12)}$ aborda em seu trabalho as atividades recreativas baseadas em esportes como um meio de melhorar a qualidade de vida de crianças e jovens da periferia, em seu estudo ele aponta como as atividades recreativas podem atingir de melhor forma a saúde física e psicológica desses indivíduos. Estudos trazidos pelos autores demonstram como essas práticas realizadas em grupos são eficazes para a adesão dos jovens. E quando tratamos de crianças em uma situação socioeconomicamente desfavorável a prática de qualquer esporte ou exercício se torna de mais difícil acesso, mas o artigo aborda as mudanças que podem ser feitas para a inserção desses indivíduos em atividades recreativas para a melhora na qualidade de vida.

Os autores trazem algumas articulações que podem ser feitas por profissionais de educação física em virtude de ajudar essa população, o primeiro ponto se volta para as intervenções gratuitas as atividades devem ser oferecidas de maneira acessível assim como deve ser possível adaptar as instalações para que seja próximo do público alvo. As atividades também devem ser adaptadas para que todos possam participar. Com isso o impacto dessas atividades recreativas na comunidade pode ser muito duradouro oferecendo uma melhor saúde física e mental ${ }^{(12)}$.

Como o fator das relações pessoais e sociais afetam a saúde cognitiva e mental dos alunos e qual a influência da atividade física para o desenvolvimento desses fatores.

Sobre as questões interpessoais, Veiga et al. ${ }^{(15)}$ ilustra em seu trabalho como as relações de apoio familiar podem influenciar os aspectos comportamentais de seus filhos. Nos estudos abordados pelos autores é possível observar que a participação e o envolvimento deles com as atividades escolares de seus filhos os motivaram positivamente e influenciavam em sua aprendizagem.

Veiga et al ${ }^{(16)}$ também aborda as questões socioeconômicas e socioculturais como fatores muito importantes para o comprometimento dos estudantes com seus estudos. O aspecto da qualidade de vida decai junto aos dois fatores já citados, devido ao estilo de vida que os indivíduos levam por conta da diferente realidade. Isso demonstra uma relação do desempenho acadêmico com a motivação e autoconfiança de jovens, e o resultado corrobora com os estudantes que possuem pais com uma alta escolaridade, os quais mantiveram vínculos com a escola junto ao filho e o apoiaram. 
Constituindo uma relação com o estudo de Elsborg et al ${ }^{(12)}$, no qual ele constata que indivíduos socioeconomicamente desfavorecidos possuem uma dificuldade muito maior de ter contato com qualquer prática de atividades físicas, podemos compreender portanto que os indivíduos citados nos estudos debatidos por Veiga et al ${ }^{(16)}$ também podem vir a sofrer com o fator de inatividade física e por consequência adquirir problemas sociais, físicos e psicológicos.

\section{CONCLUSÃO}

A literatura mostrou que as atividades físicas podem influenciar as crianças e jovens de maneira muito positiva. Foi possível identificar em diversos artigos a relação positiva entre a prática de atividades físicas e aspectos psicológicos dos estudantes, o que, por consequência, contribui para o desenvolvimento dos alunos, especialmente do perfil social, melhorando seu relacionamento consigo e com os demais.

Percebeu-se a importância do profissional de educação física, primeiro quando se trata de identificar essas dificuldades que tangem a vivência escolar dessas crianças e jovens, assim como para intervir com atividades físicas que visam contribuir para a saúde dos alunos em todos os âmbitos, saúde física, mental, psicológica e social.

\section{REFERÊNCIAS}

1. Ramires VRR, Passarini DS, Flores GG, dos Santos LG. Fatores de risco e problemas de saúde mental de crianças. Arq Bras Psicol. 2009;61(2):1-14.

2. Lopes J, Neto C. Saúde mental e níveis de atividade física de crianças escolares. 2017;(March).

3. Simões NC, Castro PF de. Avaliação Psicológica em Escolares: Relação entre Personalidade, Autoconceito e Habilidades Sociais. Gerais Rev Interinstitucional Psicol. 2018;11(1):26-44.

4. Gaia JWP, Ferreira RW, Pires DA. Efeitos da atividade física sobre os estados de humor de jovens estudantes. J Phys Educ ISSN 2448-2455. 2021;32(1).

5. Lubans D, Richards J, Hillman C, Faulkner G, Beauchamp M, Nilsson M, et al. Physical Activity for Cognitive and Mental Health in Youth: A Systematic Review of Mechanisms. Pediatrics [Internet]. 2016 Sep 1;138(3):e20161642. Available from: http://pediatrics. aappublications.org/content/138/3/e20161642. abstract

6. Poirel E. Psychological benefits of physical activity for optimal mental health. Sante Ment Que [Internet]. 2017;42(1):147-64. Available from: http:// europepmc.org/abstract/MED/28792566

7. Vin P, Silva C, Jr LC. [ T ] Efeitos da atividade física para a saúde de crianças e adolescentes The effects of physical activity on the health of children and adolescents [A ]. 2011;29(64):41-50.

8. Ferreira VR, Jardim TV, Póvoa TIR, Viana RB, Sousa ALL, Jardim PCV. Inatividade física no lazer e na escola está associada à presença de transtornos mentais comuns na adolescência. Rev Saude Publica. 2020;54:128.

9. Whittemore $\mathrm{R}$, Knafl $\mathrm{K}$. The integrative review: updated methodology. J Adv Nurs. 2005;52(5):546-53.

10. Kawabata M, Chua KL, Chatzisarantis NLD. A school-based intervention program in promoting leisuretime physical activity: trial protocol. BMC Public Health [Internet]. 2018;18(1):433. Available from: https://doi. org/10.1186/s12889-018-5320-1

11. Smedegaard S, Christiansen LB, Lund-Cramer P, Bredahl T, Skovgaard T. Improving the well-being of children and youths: a randomized multicomponent, school-based, physical activity intervention. BMC Public Health. 2016;16(1):1-11.

12. Elsborg P, Nielsen G, Klinker CD, Melby PS, Christensen JH, Bentsen P. Sports-based recreation as a means to address social inequity in health: why, when, where, who, what, and how. BMC Public Health [Internet]. 2019;19(1):1084. Available from: https://doi. org/10.1186/s12889-019-7428-3

13. Prodócimo E. O BRINCAR E A MANIFESTAÇÃO DAS EMOÇÕES NA ESCOLA. 2009.

14. Prodócimo E, Caetano A, de Sá CS, dos Santos FAG, Siqueira JCF. Jogo e emoções: implicações nas aulas de Educação Física Escolar. Motriz J Phys Educ UNESP. 2007;128-36.

15. Veiga FH, Robu V, Conboy J, Ortiz A, Carvalho C, Galvão D. Envolvimento dos alunos na escola e variáveis familiares: Uma revisão da literatura. Estud Psicol. 2016;33(2):187-97.

16. Veiga FH, Robu V, Conboy J, Ortiz A, Carvalho C, Galvão D. Envolvimento dos alunos na escola e variáveis familiares: Uma revisão da literatura. Estud Psicol. 2016;33(2):187-97.

OBSERVAÇÃO: Os autores declaram não existir conflitos de interesse de qualquer natureza. 